\title{
Control of Kerr Cavity Soliton Combs by Chirped Pumping
}

\author{
Francesco Rinaldo Talenti ${ }^{1, *}$, Tobias Hansson ${ }^{2}$, and Stefan Wabnitz ${ }^{1}$ \\ ${ }^{1}$ Dipartimento di Ingegneria dell'Informazione, Elettronica e Telecomunicazioni, Sapienza University of Rome, \\ 00184 Rome, Italy \\ ${ }^{2}$ Department of Physics, Chemistry and Biology, Linköping University, SE-581 83 Linköping, Sweden
}

\begin{abstract}
Pumping a dispersive Kerr cavity with chirped pump pulses permits to control the trapping position of temporal cavity soliton combs. Stability of single-cavity soliton generation is greatly enhanced by pump chirping. (c) 2020 The Author(s)
\end{abstract}

\section{Introduction}

Temporal cavity solitons (CS) are particularly interesting in the context of optical frequency comb (OFC) generation [1]. The suppression of CS interactions by means a quasi-CW and pulsed driving field was theoretically considered since long time [2]. However, experiments and theoretical modelling of micro-combs mostly refers to the case of continuous wave (CW) pumping [3]. Only recently, the idea of pulsed pumping for OCF generation has been explored [4,5]. Hendry et al. have shown that a stationary CS can be sustained by a broad Gaussian pump pulse [4].

The use of chirped pulses enables the control of $\chi^{(3)}$ phenomena: spectral broadening from self-phase modulation can be either suppressed [6] or enhanced [7] by pulse chirping. In this work, we show that chirped pulsed pumping may have an important role for the control of CS OFCs. Specifically, introducing a linear chirp permits to shift the temporal position of the CS. Moreover, chirping leads to stable, and repeatable coherent single-CS frequency combs, over a large range of pump frequency detunings from the cavity resonances.

\section{Results}

We model OFC generation based on the well-known mean-field Lugiato-Lefever equation (LLE) in a dimensionless form, including a linearly chirped pump with a super Gaussian profile:

$$
\frac{\partial E}{\partial t}=\left[-1+i\left(|E|^{2}-\Delta\right)+i \frac{\partial^{2}}{\partial \tau^{2}}\right] E+S(\tau), \quad S(\tau)=S_{0} \exp \left(-\frac{(1+i C) \tau^{2}}{2 \tau_{G}^{2}}\right)^{q},
$$

where $E$ is the field envelope, $\Delta$ is the detuning, $S$ is the pump, $t$ and $\tau$ are the slow and the fast time coordinates, respectively. At first, we consider as initial condition a CS of the form:

$$
u_{0}(\tau)=\sqrt{2 \Delta} \operatorname{sech}\left(\sqrt{\Delta}\left(\tau-\tau_{0}\right)\right) .
$$

It has been shown that such a CS can be trapped on the edge of a Gaussian pump pulse at a specific temporal position, which depends on the pump intensity [4] (see green curves in figure (1)(a,d)). By including a suitable chirp $C$, it is possible to control the relative drift velocity, hence the stable time shift between the CS and the pump pulse (see red curves in figure (1)(a,d)). We observed that, for a positive chirp $C$ above a threshold value, the CS remains aligned with the center of the pump pulse.

In fact, figure (1) shows that, depending on the initial position of the CS, the temporal shift with respect to the pump pulse may reverse its direction. This means that there is specific $C$ value, such that relative drift velocity vanishes, and the stationary CS position is the one which is set by initial conditions (see figure $(1)(\mathrm{g})$ ).

Next we considered the role of pump pulse chirping on the possibility of achieving single CS generation, which is important to obtain stable and coherent OFCs by the technique of adiabatically sweeping the cavity detuning. Our simulations in figure (2) reveal that for a positive and relatively large (e.g., $C=1$ ) linear chirp of the pulsed pump driving field, a single CS directly emerges from the modulationally unstable chaotic regime, and remains stable over a large range of variation of the cavity detuning.

In conclusion, we studied the use of chirped pumping for CS based Kerr OFC generation. We have shown that chirping permits to control the relative timing shift between the pump and the CS. In addition, a sufficiently large positive chirp leads to single CS generation with extended stability range.

This project has received funding from the European Union's Horizon 2020 Research and Innovation Programme under the Marie Skłodowska-Curie grant agreement No. 814147. 

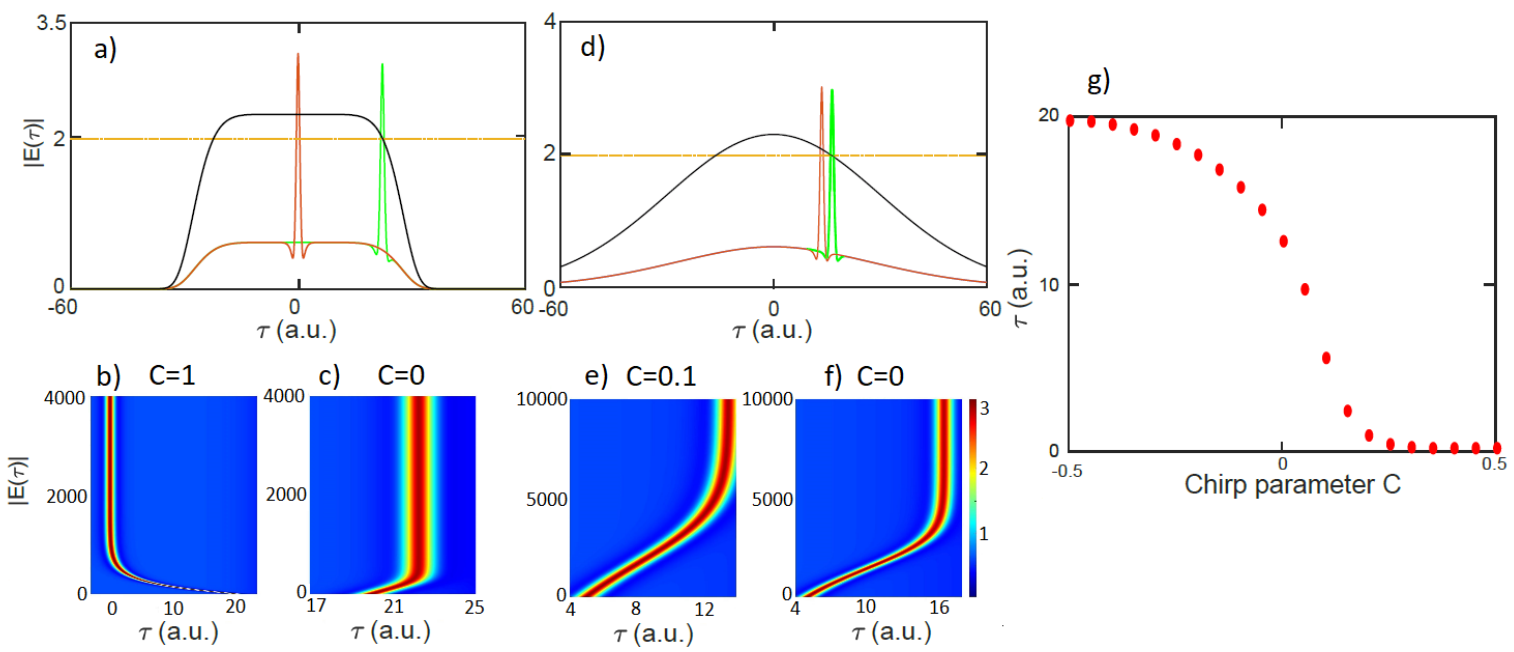

e) $\mathrm{C}=0.1$

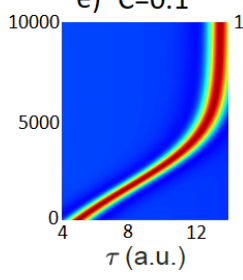

f) $C=0$

Fig. 1. In a) and d) we show the intracavity field after 4000 and 10000 round trips, respectively, for $\mathrm{C}=0$ (green line), or a) $\mathrm{C}=1, \mathrm{~d}$ ) $\mathrm{C}=0.1$ (red lines). The orange line corresponds to the critical driving strength to observe symmetry-breaking for an unchirped Gaussian pump: it determines the trapping position for the soliton in this case [4]. Panels b), c), e) f) illustrate how the CS dynamics is affected by the chirp. Here, for a), b) and c) we set $S_{0}=2.3, \tau_{g}=20, \Delta=4, q=4, \tau_{0}=20$; for d), e) and f) we set $S_{0}=2.3, \tau_{g}=20, \Delta=4, q=1, \tau_{0}=5$. In $\mathrm{g}$ ) we set the same parameter values as in d), and plot the final CS trapping position vs. $C$.
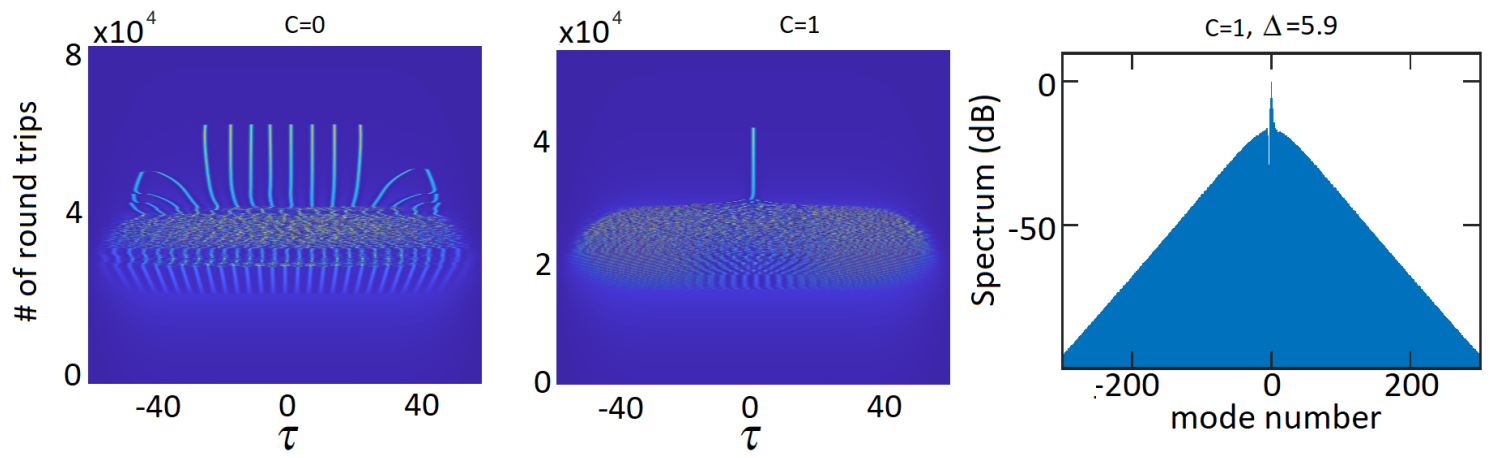

Fig. 2. The two plots on the left are the intracavity temporal intensity from adiabatic cavity detuning sweeps, for different values of the chirp parameter. We set $S_{0}=2.3, q=4, \tau_{g}=40$, and the roundtrip time of the cavity is $t_{r}=120$. On the right, the $\mathrm{OFC}$ for $\mathrm{C}=1$ and $\Delta=5.9$, corresponding to the $\sim 4 \times 10^{4}$ th round trip of the central figure.

\section{References}

1. T. Herr, et al. Temporal solitons in optical microresonators. Nature Photon 8, 145-152 (2014).

2. S. Wabnitz, Control of soliton train transmission, storage, and clock recovery by cw light injection, J. Opt. Soc. Am. B 13, $2739-2749$ (1996)

3. A. Pasquazi et al., Micro-combs: A novel generation of optical sources, Physics Reports, Volume 729, (2018).

4. I. Hendry, et al., Spontaneous symmetry breaking and trapping of temporal Kerr cavity solitons by pulsed or amplitude-modulated driving fields. Physical Review A, 97(5), (2018).

5. M. Malinowski, et al., Optical frequency comb generation by pulsed pumping. APL Photonics. 2.(2017).

6. F. Audo, et al., Nonlinear spectrum broadening cancellation by sinusoidal phase modulation" Opt. Lett. 42, 2902-2905 (2017).

7. N. Vermeulen, et al., Negative Kerr nonlinearity of graphene as seen via chirped-pulse-pumped self-phase modulation. Physical Review Applied, 6, 10 (2016). 